Article

\title{
Photocatalytic Functionalized Aggregate: Enhanced Concrete Performance in Environmental Remediation
}

\author{
Amer Hakki ${ }^{1,2, *}$, Lu Yang ${ }^{1,3}$, Fazhou Wang ${ }^{3}$, Ammar Elhoweris ${ }^{2}$, Yousef Alhorr ${ }^{2}$ and \\ Donald E. Macphee ${ }^{1, *}$ \\ 1 Department of Chemistry, University of Aberdeen, Meston Building, Meston Walk, Aberdeen AB24 3UE, \\ UK; yanglu@whut.edu.cn \\ 2 Gulf Organisation of Research and Development, Qatar Science and Technology Park, Doha 210162, Qatar; \\ a.elhoweris@gord.qa (A.E.); alhorr@gord.qa (Y.A.) \\ 3 State Key Laboratory of Silicate Materials for Architectures, Wuhan University of Technology, 122\# Luoshi \\ Road, Wuhan 430070, China; fzhwang@whut.edu.cn \\ * Correspondence: a.hakki@gord.qa (A.H.); d.e.macphee@abdn.ac.uk (D.E.M.); Tel.: +974-44215006 (A.H.); \\ +44-(0)1224-272941 (D.E.M.)
}

Received: 2 December 2018; Accepted: 4 January 2019; Published: 22 January 2019

check for updates

\begin{abstract}
Engineering of effective photocatalytically active structures is of great importance as it introduces a solution for some existing air pollution problems. This can be practically achieved through the bonding of particulate photocatalysts to the surface of construction materials, such as aggregates, with a suitable stable binding agent. However, the accessibility of the photocatalytically active materials to both the air pollutants and sunlight is an essential issue which must be carefully considered when engineering such structures. Herein, different amounts of commercial $\mathrm{TiO}_{2}$ were supported on the surface of quartz sand, as an example of aggregates, with a layer of silica gel acting as a binder between the photocatalyst and the support. The thus prepared photocatalytically active aggregates were then supported on the surface of mortars to measure their performance for NOx removal. The obtained materials were characterized by electron microscopy (SEM and TEM), Fourier Transform Infrared Spectroscopy (FTIR), X-ray Diffraction (XRD), and UV-vis Absorption Spectroscopy. Very good coverage of the support's surface with the photocatalyst was successfully achieved as the electron microscopic images showed. FTIR spectroscopy confirmed the chemical bonding, i.e., interfacial Ti-O-Si bonds, between the photocatalyst and the silica layer. The photocatalytic activities of the obtained composites were tested for photocatalytic removal of nitrogen oxides, according to the ISO standard method (ISO 22197-1). The obtained aggregate-exposed mortars have shown up to ca. four times higher photocatalytic performance towards NO removal compared to the sample in which the photocatalyst is mixed with cement, however, the nitrate selectivity can be affected by Ti-O-Si bonding.
\end{abstract}

Keywords: environmental remediation; air pollution; photocatalytic construction materials; nitric oxides; functionalized aggregate

\section{Introduction}

Air pollution is a serious problem which directly affects everyone who lives in populated cities around the world [1]. Incontrovertibly, fossil fuel combustion for energy production and in the transportation sector, together with metallurgical industries, i.e., cementitious and construction industries, are the major sources of most anthropogenically produced pollutants [2]. Common air pollutants that pose risks to human health include nitrogen oxides (NOx; mainly $\mathrm{NO}$ and $\mathrm{NO}_{2}$ ), carbon monoxide $(\mathrm{CO})$, sulfur oxides $(\mathrm{SOx})$, ozone $\left(\mathrm{O}_{3}\right)$, volatile organic compounds (VOCs), and 
particulate matter (PM) [3]. Among them, NOx gases have attracted a lot of concern since they cause or worsen serious health hazards for humans, including diseases such as emphysema and bronchitis, and aggravate existing heart disorders. Moreover, NOx gases are responsible for acid rains and smog and contribute to greenhouse warming [4].

Under solar irradiation, some photocatalysts such as $\mathrm{TiO}_{2}$ have the potential to reduce ambient concentrations of NOx from areas in which the concentrations of these pollutants exceed national exposure level guidelines [5]. The light-induced oxidation reactions occur on the surface of irradiated $\mathrm{TiO}_{2}$ in the presence of molecular oxygen and convert the toxic $\mathrm{NO}$ gas into nitrate through subsequent oxidation steps, with the intermediate formation of nitrogen dioxide: $\mathrm{NO} \rightarrow \mathrm{NO}_{2} \rightarrow \mathrm{NO}_{3}{ }^{-}$[6-9]. The thus formed nitrate $\left(\mathrm{NO}_{3}{ }^{-}\right)$on the $\mathrm{TiO}_{2}$ surface can be rinsed and the catalyst can be recovered for further photocatalytic cycles.

Because of their high surface area and their direct contact with urban atmospheres, construction materials are excellent carriers to which photocatalytic materials can be applied for air purification. Recently, photocatalytically active surfaces have established an important commercial as well as technological position in modern construction technologies [10]. Some trials on the application of $\mathrm{TiO}_{2}$ photocatalysts in several concrete applications have already been conducted. Richard Meier's Dives in Misericordia Church project in Rome [11], the New Road Construction Concepts (NR2C), the Air Quality Innovation Programme (IPL) in the Netherlands, and Photocatalytic Innovative Coverings Applications for Depollution (PICADA) are examples of such trials and projects [5]. These approaches have typically focused on the addition of the photocatalytic materials to the binder phase, where their performance can be strongly influenced by poor dispersibility or occlusion of the added photocatalytic powder [12-14]. Moreover, some reported studies address the impact of photocatalyst additions on the practical characteristics of concrete, e.g., workability and durability [15]. Despite our level of understanding of these mechanisms, there have been few innovative solutions to maintain the cement-free photocatalyst performance level over time.

Therefore, engineering photocatalytically active structures in a way that ensures high performance in air purification is of great importance from both environmental and economical perspectives [16-18]. The approach suggested in this work is supportive of $\mathrm{TiO}_{2}$-based photocatalysts on aggregate materials such that the photocatalyst is positioned above the surface of the concrete. Placement of such aggregates on the surface of the concrete structure during construction ensures that the photocatalyst will have free access to atmospheric pollutants (particularly NOx), rainwater and illuminating radiation, and be free of agglomeration issues. However, although this approach has been previously reported, efficient binding of photocatalytic material to the support surface is still a challenging issue.

\section{Materials and Methods}

\subsection{Synthesis of $\mathrm{TiO}_{2}-\mathrm{SiO}_{2}-\mathrm{Quartz}$ Composites}

Standard quartz sand $(\mathrm{Q})$ was first sieved to obtain a sample with the desired particle size distribution (1-3 mm) before it was washed with deionized water several times and dried at $95{ }^{\circ} \mathrm{C}$. A commercial photocatalyst (CristalACTiV ${ }^{\mathrm{TM}}$ PC105, Cristal, Stallingborough, UK) was supported on the obtained $\mathrm{Q}$ using a silica-based binder prepared from the precursor tetraethyl orthosilicate (TEOS, Sigma-Aldrich, Schnelldorf, Germany). A TEOS mother solution was prepared by adding the exact amount of TEOS into an ethanol:water: $\mathrm{HCl}$ mixture at a TEOS:ethanol:water: $\mathrm{HCl}$ molar ratio of 1:3:4:4 $\times 10^{-3}$, which was stirred for 10 days at room temperature in a sealed vessel [19]. Then the required volumes of the obtained solution were added to $200 \mathrm{~mL}$ of ethanol, in which the required amounts of $\mathrm{TiO}_{2}$ were suspended, to get $0.5,1,2.5,5$, and $10 \mathrm{wt} \% \mathrm{TiO}_{2}$ :Quartz in the final product. These values were chosen because many reports identified the efficacy of $\mathrm{TiO}_{2}$ photocatalysts conventionally mixed into cementitious materials (with $\mathrm{TiO}_{2}$ loadings from 1 to $10 \mathrm{wt} \%$ as a fraction of the cement content) [20-22]. The molar ratio of $\mathrm{TiO}_{2}: \mathrm{TEOS}$ was always kept at 1:1. The suspension was then kept gently stirring at room temperature overnight. It was then added dropwise to $40 \mathrm{~g}$ of $\mathrm{Q}$ 
with continuous stirring at $80^{\circ} \mathrm{C}$ under reduced pressure. The obtained materials were dried at $90{ }^{\circ} \mathrm{C}$ overnight followed by heat treatment at $200{ }^{\circ} \mathrm{C}$ for $4 \mathrm{~h}$. The thus obtained modified $\mathrm{Q}$ samples were then cooled in air, washed with deionized water, dried at $90^{\circ} \mathrm{C}$, and sieved again to collect particles larger than $1 \mathrm{~mm}$ in order to separate modified quartz from loosely or non-connected $\mathrm{TiO}_{2}$-binder. The resulting composite samples are denoted QTx where $x$ represents expected $\mathrm{TiO}_{2}$ content. In order to simplify the characterisation, fine powdered Quartz (particles sizes in the range 20-50 $\mu \mathrm{m}$ obtained by ball milling of commercial quartz (Aldrich) and sieving) was also modified with $10 \mathrm{wt} \% \mathrm{TiO}_{2}$ following a similar procedure; the obtained sample is denoted FQT.

\subsection{Characterization}

X-ray diffraction (XRD) patterns were obtained using a PANalytical diffractometer $\left(X^{\prime}\right.$ Pert $^{3}$ Powder, Malvern Panalytical, UK) equipped with a CuKa1 $1.54 \AA$ X-ray source. FTIR spectra were recorded using a PerkinElmer Spectrum Two equipped with UATR (Single Reflection Diamond, PerkinElmer, Inc, City, State, USA). UV-vis diffuse reflectance spectra of the samples were recorded using a Cary 60 UV-vis spectrophotometer (Agilent Technology, City, State, USA) equipped with a fibre optic coupler. Barium sulphate was used as a reference in the range of 250 to $600 \mathrm{~nm}$. The resulting reflectance spectra were transformed into apparent absorption spectra by using the Kubelka-Munk function $\mathrm{F}(\mathrm{R} \infty)=(1-\mathrm{R} \infty)^{2} / 2 \mathrm{R} \infty$. The amount of $\mathrm{TiO}_{2}$ loaded on the surface of $\mathrm{Q}$ was analyzed by $\mathrm{X}$-ray fluorescence spectroscopy (XRF) using Rigaku NexQC. For this purpose, a calibration series of $\mathrm{TiO}_{2}-\mathrm{SiO}_{2}$ was prepared by mixing the required amount of $\mathrm{TiO}_{2}(\mathrm{PC} 105)$ and $\mathrm{SiO}_{2}$ (ball milled commercial quartz) to get a series from 0.1 to $20 \mathrm{wt} \% \mathrm{TiO}_{2}$. Morphologies of samples were observed using a scanning electron microscope (SEM, Zeiss EVO MA10, Zeiss, City, State, USA) equipped with an energy dispersive $\mathrm{X}$-ray spectrometer (EDS, Oxford INCA) for elemental composition analyses. The transmission electron microscopy (TEM) was performed on a JEOL-JEM-2000EX microscope operated with an accelerating voltage of $200 \mathrm{kV}$; images were captured with a Gatan Erlangshen ES500W camera.

\subsection{Photocatalytic Performance Test}

The removal of nitrogen oxides (NOx), as examples of air pollutants, using the obtained quartz sand modified with the photocatalyst was tested to check their efficiency in purification of the polluted air [23]. Figure 1 illustrates the air-purification test set-up which was established for this purpose. The set-up consists of the following (see Figure 1): gas suppliers; i.e., synthetic air and NO in nitrogen (BOC, Guildford, UK), gas flow controllers (Bronkhorst, Newmarket Suffolk, UK) (1), a humidity supplier unit (2), a photocatalytic reactor according to ISO standard design (ISO 22197-1) (3), a UV(A) irradiation source (4), and a NOx analyzer (5). The gas supplies were NO (100 ppm) in $\mathrm{N}_{2}$ and synthetic air (BOC, UK). The required concentration (1000 ppbv), flow rates $\left(5 \times 10^{-5} \mathrm{~m}^{3} \cdot \mathrm{s}^{-1}\right)$, and humidity (ca. $40 \%$, confirmed by a Rotronic hygropalm) were achieved by controlling the flow of each gas by the gas flow controllers (1). The photoreactor was constructed from PMMA (Poly(methyl methacrylate)), covered with borosilicate glass, and was positioned below the output from an $S S 0.5 \mathrm{~kW}$, $500 \mathrm{~W}$ fully reflective solar simulator equipped with a 1.5 AM filter (Sciencetech, London, Ontario, Canada). The irradiation was adjusted to ensure that the test sample (6) received a light intensity of $10 \mathrm{Wm}^{-2}$ at $\lambda<420 \mathrm{~nm}$. The light intensity was measured using a broadband thermopile detector (Gentec-EO-XLP12-3S-H2-D0) located exactly where the test sample should be placed. A Thermo Scientific Model 42i-HL High Level NO-NO $\mathrm{N}_{2}-\mathrm{NOx}$ Analyzer (Air Monitors Ltd., Gloucestershire, UK) was used to monitor the concentrations of $\mathrm{NO}, \mathrm{NO}_{2}$, and total $\mathrm{NOx}$ in the outlet gas flow. Aggregate exposed mortars were prepared by supporting $10 \mathrm{~g}$ of bare or $\mathrm{TiO}_{2}$-modified quartz sand on the top of mortars (with top surface area of $64 \mathrm{~cm}^{2}$ and thickness of $15 \mathrm{~mm}$ ) in order to examine their efficiencies in NOx removal. For comparison, a reference sample was prepared according to the conventional mixing method (CMM) in which $\mathrm{TiO}_{2}$ was first mixed with cement at a $10 \mathrm{wt} \%$ ratio. Once prepared, 
a $2 \mathrm{~mm}$ thick layer of the mortar mixture was applied to the top of the pre-made mortar similar to those used to prepare the aggregate exposed mortars.

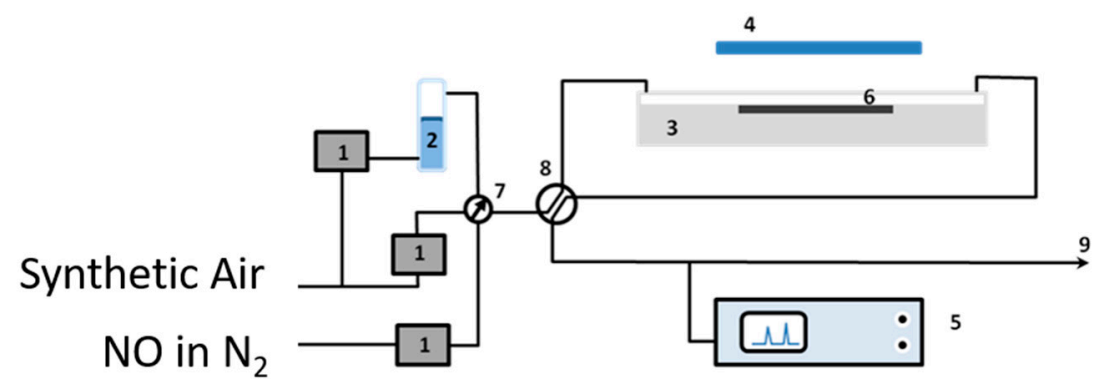

Figure 1. The photocatalytic testing apparatus: (1) mass flow controllers, (2) humidity supplier, (3) photocatalytic reactor, (4) UV(A) irradiation source, (5) NOx analyzer, (6) test sample, (7) and (8) valves, and (9) gas stream outlet.

\section{Results and Discussion}

XRD patterns of uncoated quartz sand $(\mathrm{Q})$ and the prepared $\mathrm{TiO}_{2}-\mathrm{SiO}_{2}-\mathrm{Quartz}$ composite (FQT) are shown in Figure 2. The appearance of a clear XRD pattern for anatase $\mathrm{TiO}_{2}$ in FQT sample confirms the successful support of $\mathrm{TiO}_{2}$ on the quartz in FQT.

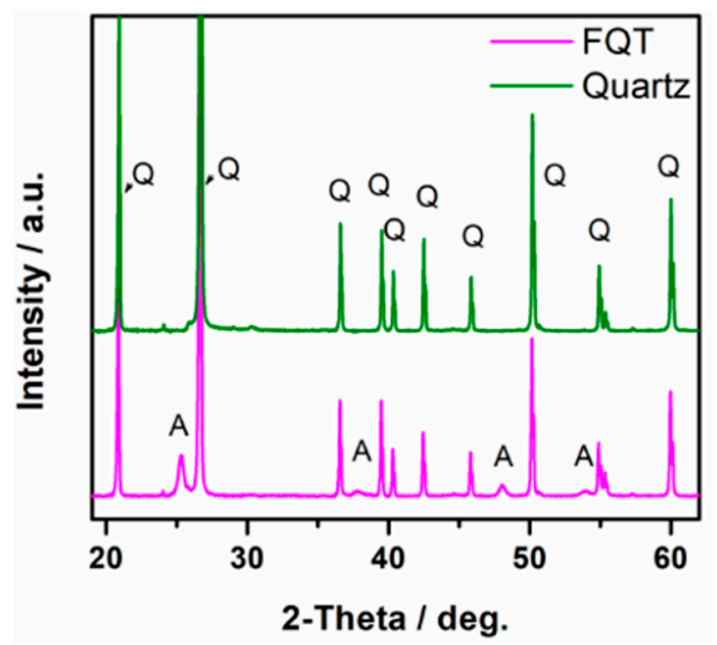

Figure 2. XRD patterns of bare quartz and $\mathrm{TiO}_{2}-\mathrm{SiO}_{2}-$ Quartz composite (FQT), $\mathrm{Q}$ and A refer to quartz and anatase patterns, respectively.

Evidence for the formation of a chemical bond between the $\mathrm{TiO}_{2}$ and $\mathrm{SiO}_{2}$ binding layers can clearly be observed from the FTIR spectrum in the range between $900-960 \mathrm{~cm}^{-1}$ (Figure 3), the absorption being assigned to the stretching vibrational mode of the Ti-O-Si bond. No similar absorption peak due to this mode was observed for $\mathrm{SiO}_{2}$ in the bare $\mathrm{Q}$ sample. 


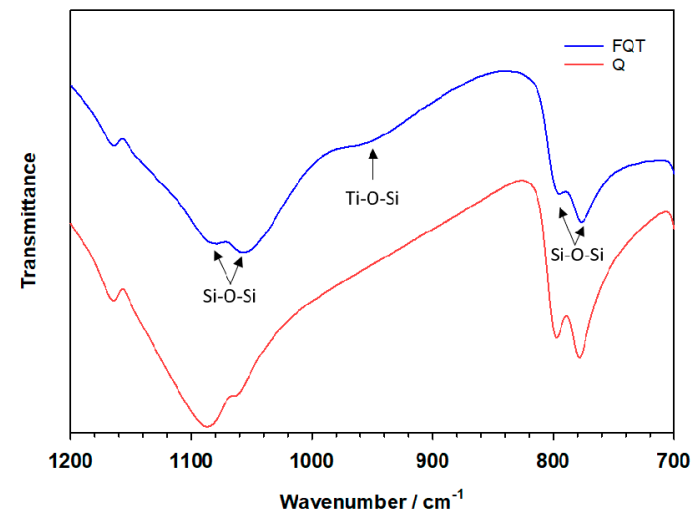

Figure 3. FTIR spectra of bare quartz $(\mathrm{Q})$ and the $\mathrm{TiO}_{2}-\mathrm{SiO}_{2}-$ Quartz composite (FQT).

Figure 4 shows the diffuse reflectance UV-vis absorption spectra of free $\mathrm{TiO}_{2}$ and $\mathrm{FQT}$ composite samples. Considering $\mathrm{TiO}_{2}$ as an indirect semiconductor, the modified Kubelka-Munk function $[\mathrm{F}(\mathrm{R} \infty) \mathrm{h} v]^{1 / 2}$ was plotted as a function of the incident photon energy which allowed the determination of the photocatalyst's band gap. The spectra are additional evidence for the presence of $\mathrm{TiO}_{2}$ in the FQT sample and show that the loading of $\mathrm{TiO}_{2}$, via the herein employed method, on the surface of quartz through $\mathrm{SiO}_{2}$ binding layer has negligible effect on its band gap.

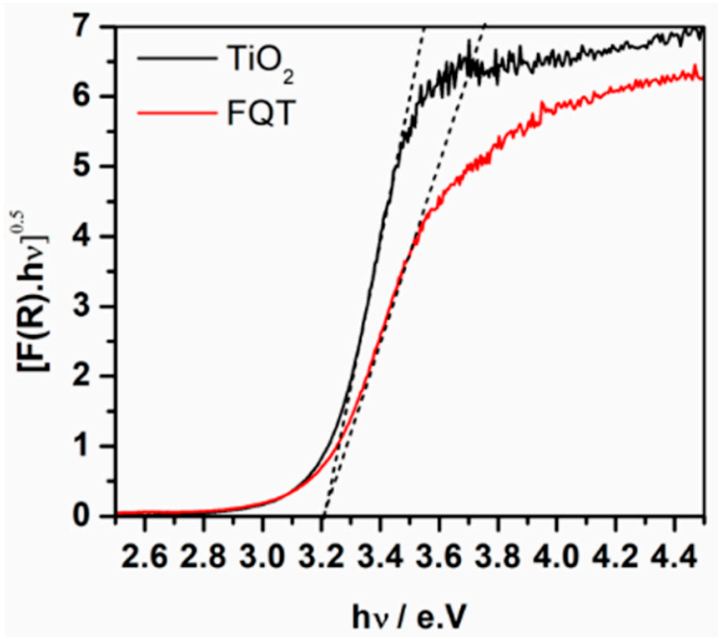

Figure 4. UV-vis absorption spectra presented as the modified Kubelka-Munk function of free $\mathrm{TiO}_{2}$ and $\mathrm{TiO}_{2}-\mathrm{SiO}_{2}-$ Quartz composite (FQT).

The amounts of $\mathrm{TiO}_{2}$ loaded on the surface of $\mathrm{Q}$, as recorded by XRF analyses, are summarized in Table 1. As can be seen from this table, the actual amount of $\mathrm{TiO}_{2}$ loaded is lower than the calculated values for all the prepared samples, meaning that some loss of $\mathrm{TiO}_{2}$ occurred during the preparation procedure. However, the lower the loaded amount the lower the $\mathrm{TiO}_{2}$ loss. This indicates a limitation of the modifying layer thickness over which all the extra added amount of $\mathrm{TiO}_{2}$-binder is lost.

Table 1. Calculated and analyzed loaded amount of $\mathrm{TiO}_{2}$ on quartz via silica gel binder.

\begin{tabular}{cccc}
\hline \multirow{2}{*}{ Sample ID } & \multicolumn{2}{c}{$\mathrm{TiO}_{\mathbf{2}} \mathbf{( w t}$ \%) } & \multirow{2}{*}{$\mathrm{TiO}_{\mathbf{2}}$ Loss (\%) } \\
\cline { 2 - 3 } & Calculated & Analyzed & \\
\hline QT0.5 & 0.5 & 0.35 & 30 \\
QT1 & 1.0 & 0.63 & 37 \\
QT2.5 & 2.4 & 0.72 & 70 \\
QT5 & 4.7 & 2.9 & 38 \\
QT10 & 9.1 & 2.67 & 71 \\
\hline
\end{tabular}


The level of coverage of the support $(\mathrm{Q})$ with $\mathrm{TiO}_{2}$ was examined using the SEM-EDS technique. Figure 5 compares the SEM-EDS of bare quartz coated with a silicate layer derived from TEOS in which commercial $\mathrm{TiO}_{2}$ (PC105) is dispersed. The effectiveness of a silicate-based layer on quartz (FQT) for the efficient support of $\mathrm{TiO}_{2}$ can be clearly seen from these SEM images. Very good coverage of $\mathrm{TiO}_{2}$ over the sample is achieved as a result of coating a silicate-based film over the quartz substrate.
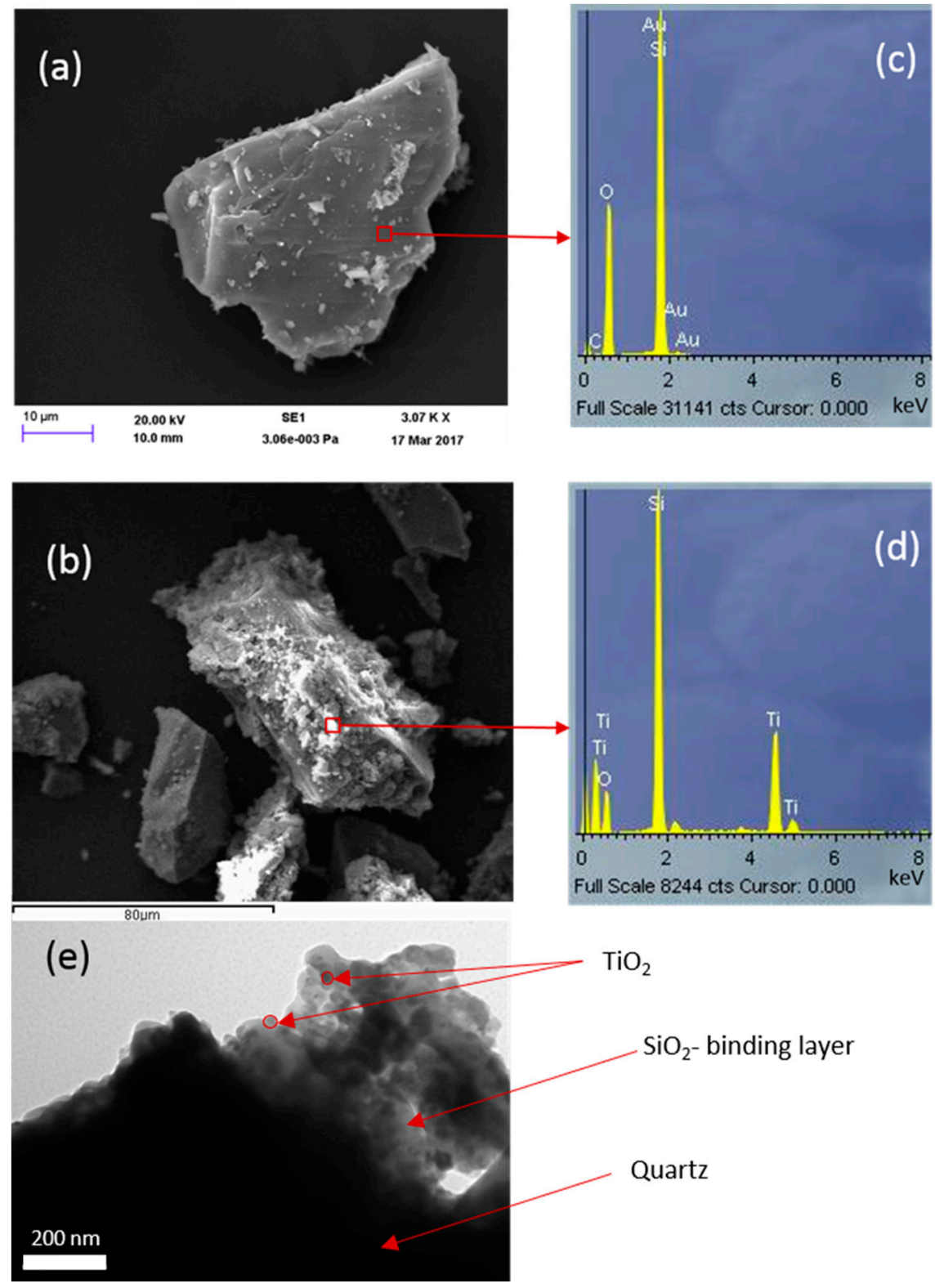

Figure 5. SEM images for (a) uncoated quartz and (b) $\mathrm{TiO}_{2}$ (commercial PC105) immobilized on quartz via a $\mathrm{SiO}_{2}$ binder formed from tetraethyl orthosilicate (TEOS). EDS of these samples are presented in $(\mathbf{c}, \mathbf{d})$, and the TEM image for the same samples in (e).

Although a few areas might be clear of silicate coating, Figure 5 shows that the silicate layer has generally been immobilized nicely on the grains. Consequently, in this case, $\mathrm{TiO}_{2}$, associated with the silicate-based gel phase, is also similarly distributed and is not bonded directly to the quartz surface. This is consistent with the TEM image in Figure 5e. Comparing the EDS analyses (Figure $5 c, d$ ) indicates the spreading of $\mathrm{TiO}_{2}$ with the silicate layer.

In order to examine the effectiveness of the obtained modified quartz sand samples for the removal of nitrogen oxides from polluted air, aggregate exposed mortars (with top surface areas of 
$64 \mathrm{~cm}^{2}$ ) were prepared, on top of which $10 \mathrm{~g}$ of bare or $\mathrm{TiO}_{2}$-modified quartz sand were supported. An image of the obtained mortars is presented in Figure 6 which shows a clear change in the color of the quartz sand to a whiter one by increasing the amounts of loaded $\mathrm{TiO}_{2}$.

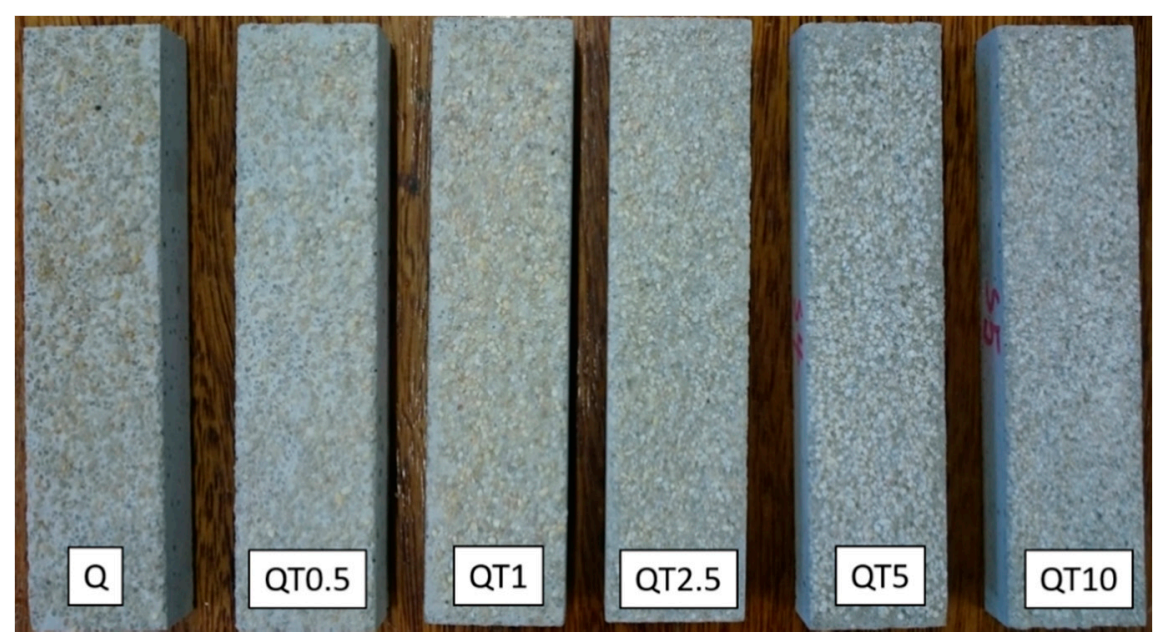

Figure 6. Images of the prepared aggregate exposed mortars. On the top of each sample, $10 \mathrm{~g}$ of bare (Q) or $\mathrm{TiO}_{2}$-modified quartz sand (QTx) were supported.

Figure 7 shows an example of the changes in the concentrations of nitrogen oxides, i.e., NO, $\mathrm{NOx}$, and $\mathrm{NO}_{2}$, in the gas stream flows over the QT10 sample in the dark and under irradiation. The concentration of NO is constant at ca. $1000 \mathrm{ppb}$ as long as the light is off. When the light is switched on, the initial $\mathrm{NO}$ concentration drops with a simultaneous increase in $\mathrm{NO}_{2}$ concentration, as $\mathrm{NO}_{2}$ is one of the NO oxidation products. Consequently, the concentration of NOx, which reflects the total oxidation of $\mathrm{NO}$ to $\mathrm{HNO}_{3}$, is reduced during the illumination time.

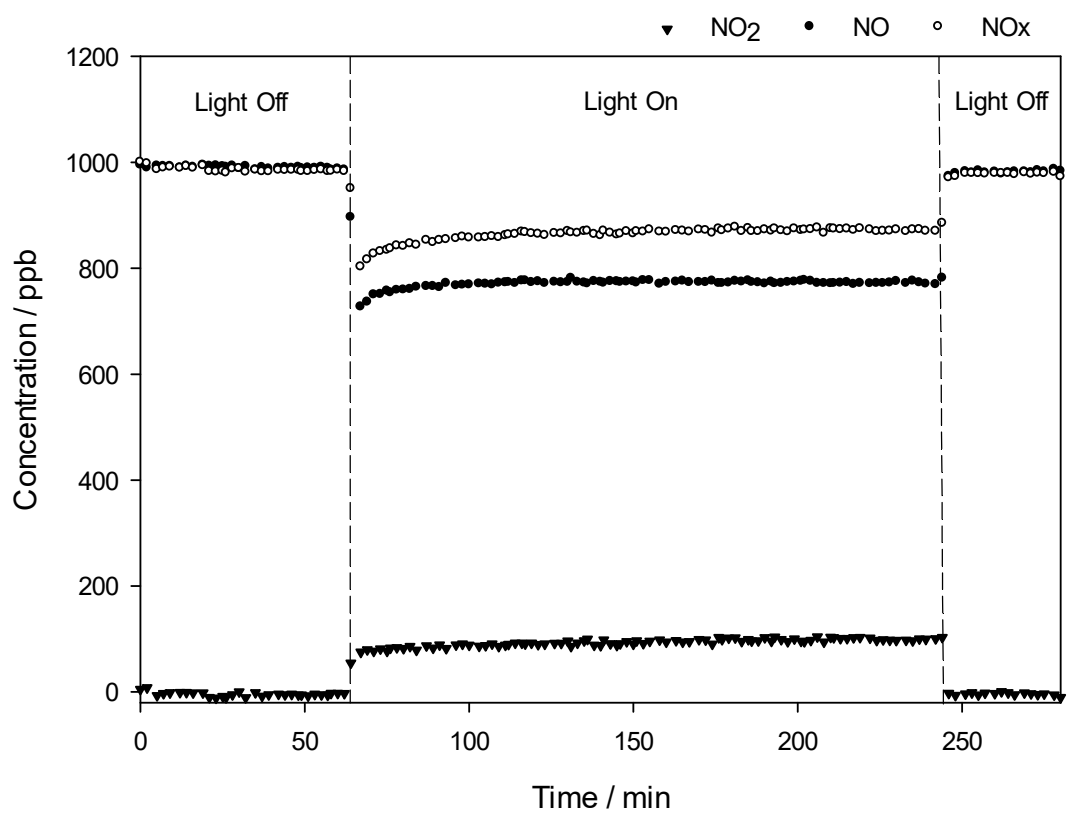

Figure 7. Changes in the concentrations of nitrogen oxides, i.e., $\mathrm{NO}, \mathrm{NO}_{2}$, and $\mathrm{NOx}$, as a function of time in the presence of the QT10 sample.

The activities of the obtained samples for De-NOx remediation were determined by measuring the photonic efficiencies $(\xi)$, which is defined as the ratio of the reaction rate (NO and NOx removal as well as $\mathrm{NO}_{2}$ formation) and the incident photon flux [24]. Equation (1) was used to calculate $\xi$ [25], where 
$\dot{V}$ is the volumetric flow rate, $\mathrm{c}_{\mathrm{d}}$ the concentration of each of the nitrogen oxide gases recorded under dark conditions, $c_{i}$ the concentration of the same gases recorded under illumination, $p$ the pressure, $\mathrm{N}_{\mathrm{A}}$ the Avogadro constant, $\mathrm{h}$ the Plank constant, $\mathrm{c}$ the speed of light, I the incident irradiation intensity, $\lambda$ the employed wavelength assuming monochromatic light $(365 \mathrm{~nm})$, A the irradiated area, $R$ the gas constant, and $\mathrm{T}$ the absolute temperature. The obtained results are illustrated in Figure 8a. The results of nitrate selectivity, which was calculated according to Equation (2) employing the obtained $\xi \mathrm{NO}$ and $\xi \mathrm{NO}_{2}$, both measured on the same sample, are shown in Figure 8b.

$$
\begin{gathered}
\xi=\frac{\dot{\mathrm{V}}\left(\mathrm{c}_{\mathrm{d}}-\mathrm{c}_{\mathrm{i}}\right) \mathrm{pN}_{\mathrm{A}} \mathrm{hc}}{\mathrm{I} \lambda \mathrm{ART}} \\
\mathrm{S}=\frac{\xi_{\mathrm{NOx}}}{\xi_{\mathrm{NO}}}
\end{gathered}
$$
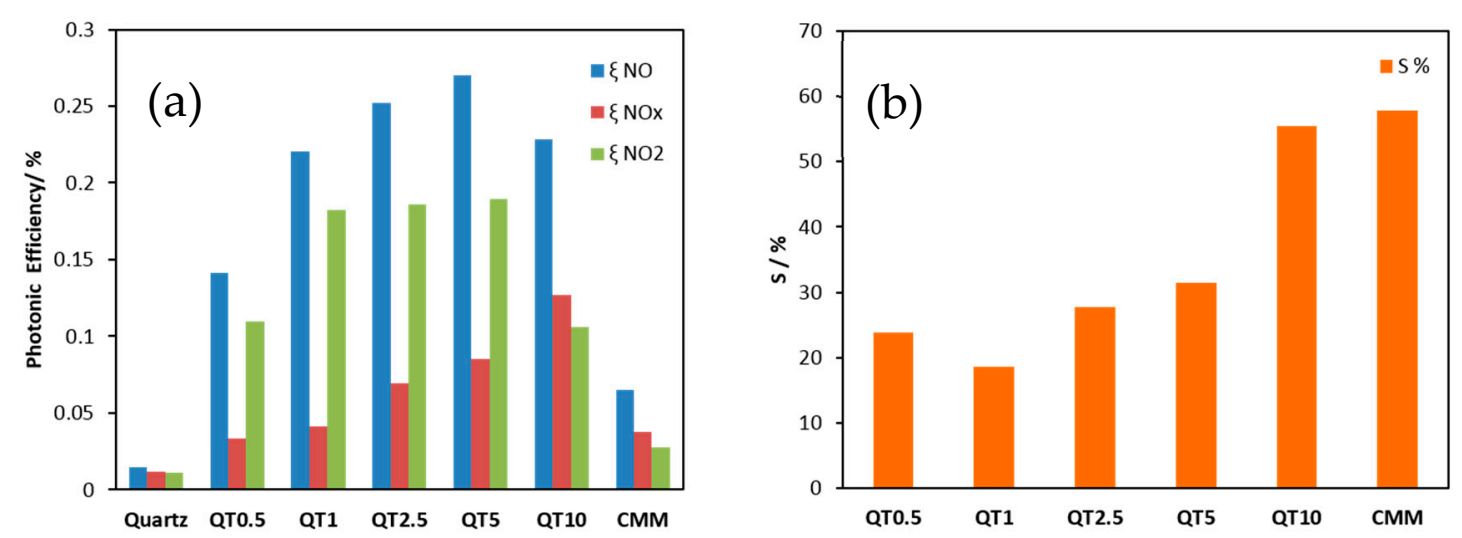

Figure 8. Photonic efficiencies of the aggregate exposed mortars prepared from bare quartz or $\mathrm{TiO}_{2}$-modified quartz for $\mathrm{NO}$ and $\mathrm{NOx}$ removal and for $\mathrm{NO}_{2}$ formation (a), and nitrate selectivity recorded for the same samples $(\mathbf{b})$.

As can be seen from Figure $8 \mathrm{a}$, the De-NOx performance $(0.14-0.23 \%$ z NO) is clearly enhanced by supporting the photocatalyst on quartz sand when compared to its performance $(0.06 \% \xi \mathrm{NO})$ when mixed with cement (CMM). This means that supporting $\mathrm{TiO}_{2}$ on the quartz surface via a silica gel binder ensures a much higher effective surface area which is translated into higher photocatalytic efficiency. It is worth noting that the herein recorded photonic efficiency for NO oxidation measured for $\mathrm{TiO}_{2}$ supported on quartz (the QT5 sample for example) is about $58 \%$ of that reported in literature for the highly active bare anatase $\mathrm{TiO}_{2}$ (Hombikat UV100) [25]. Bearing in mind that QT5 had only $2.9 \%$ of the $\mathrm{TiO}_{2}$ loaded, a significantly efficient utilization of the valuable photocatalyst is consequently achieved by supported systems. It is also worth mentioning that although the De-NOx performance improves by increasing the amount of photocatalyst loaded from QT1 to QT5, QT1 might be preferred from an economical viewpoint as this sample showed a $\xi$ NO of more than $80 \%$ of that measured for QT5 although QT1 had ca. five times less $\mathrm{TiO}_{2}$ compared to QT5. On the other hand, nitrate selectivity (Figure $8 \mathrm{~b}$ ) was also enhanced by increasing the amount of photocatalyst loaded but did not exceed that of CMM. The high selectivity is surely a valuable opportunity and improved by a higher loading amount. This observation can be explained by the fact that the porous silica gel coating presents a support for $\mathrm{TiO}_{2}$ both as $\mathrm{TiO}_{2}$ bonded to the silica gel pore walls (which usually negatively affects the selectivity) but also as $\mathrm{TiO}_{2}$ trapped in the pores. This non-bonded $\mathrm{TiO}_{2}$ offers increased activity and selectivity. Factors which control nitrate selectivity appear to be complex. Relevant variables include $\mathrm{TiO}_{2}$ polymorphism, defect state, availability of water, etc., but the role of substrate binding must also be considered. 


\section{Conclusions}

$\mathrm{TiO}_{2}$ has been successfully loaded on the surface of aggregate, with quartz used as an example, utilizing a silicate-based binder. Aggregate exposed mortars with these photocatalytically active aggregates on the surface have shown an excellent performance at the photocatalytic removal of NO from polluted air. The herein developed aggregate exposed mortars have shown up to ca. four times higher efficiency for NO removal compared with the sample prepared according to the conventional mixing method (CMM). Thus, the herein prepared supported $\mathrm{TiO}_{2}$ aggregates offer much greater utilization efficiency for the valuable photocatalyst and thus correspond to a considerably lower cost of use in photocatalytic concrete.

Author Contributions: Conceptualization, A.H. and D.E.M.; methodology, A.H. and L.Y.; validation, A.H., L.Y. and D.E.M.; formal analysis, A.H.; investigation, A.H.; resources, D.E.M. and F.W.; data curation, A.H.; writing - original draft preparation, A.H.; writing—review and editing, D.E.M., A.E. and Y.A.; visualization, A.H., D.E.M. and A.E.; supervision, D.E.M. and F.W.; project administration, D.E.M.; funding acquisition, D.E.M. and F.W.

Funding: This research was funded by the UK Engineering and Physical Sciences Research Council (Grant Ref: EP/M003299/1) and the Natural Science Foundation of China (No. 51461135005) International Joint Research Project (EPSRC-NSFC). The APC was funded by (GORD).

Acknowledgments: The authors gratefully acknowledge funding from the UK Engineering and Physical Sciences Research Council (Grant Ref: EP/M003299/1) and the Natural Science Foundation of China (No. 51461135005) International Joint Research Project (EPSRC-NSFC). L. Zheng and M. R. Jones from the Division of Civil Engineering, University of Dundee, Dundee, UK are gratefully acknowledged for the preparation of the mortars.

Conflicts of Interest: The authors declare no conflict of interest.

\section{References}

1. West, J.J.; Cohen, A.; Dentener, F.; Brunekreef, B.; Zhu, T.; Armstrong, B.; Bell, M.L.; Brauer, M.; Carmichael, G.; Costa, D.L.; et al. What We Breathe Impacts Our Health: Improving Understanding of the Link between Air Pollution and Health. Environ. Sci. Technol. 2016, 50, 4895-4904. [CrossRef] [PubMed]

2. Walton, H.; Dajnak, D.; Beevers, S.; Williams, M.; Watkiss, P.; Hunt, A. Understanding the Health Impacts of Air Pollution in London. King's College London, 15 July 2015.

3. WHO. Ambient (Outdoor) Air Quality and Health; WHO: Geneva, Switzerland, 2014.

4. Abdul-Wahab, S.A.; Chin Fah En, S.; Elkamel, A.; Ahmadi, L.; Yetilmezsoy, K. A review of standards and guidelines set by international bodies for the parameters of indoor air quality. Atmos. Pollut. Res. 2015, 6, 751-757. [CrossRef]

5. Serpone, N. Heterogeneous Photocatalysis and Prospects of $\mathrm{TiO}_{2}$-Based Photocatalytic DeNOxing the Atmospheric Environment. Catalysts 2018, 8, 553. [CrossRef]

6. Engel, A.; Große, J.; Dillert, R.; Bahnemann, D.W. The Influence of Irradiance and Humidity on the Photocatalytic Conversion of Nitrogen(II) Oxide. J. Adv. Oxid. Technol. 2015. [CrossRef]

7. Devahasdin, S.; Fan, C.; Li, K.; Chen, D.H. TiO 2 photocatalytic oxidation of nitric oxide: Transient behavior and reaction kinetics. J. Photochem. Photobiol. A Chem. 2003, 156, 161-170. [CrossRef]

8. Maggos, T.; Bartzis, J.G.; Liakou, M.; Gobin, C. Photocatalytic degradation of NOx gases using $\mathrm{TiO}_{2}$-containing paint: A real scale study. J. Hazard. Mater. 2007, 146, 668-673. [CrossRef] [PubMed]

9. Hüsken, G.; Hunger, M.; Brouwers, H.J.H. Experimental study of photocatalytic concrete products for air purification. Build. Environ. 2009, 44, 2463-2474. [CrossRef]

10. Ballari, M.M.; Brouwers, H.J.H. Full scale demonstration of air-purifying pavement. J. Hazard. Mater. 2013, 254-255, 406-414. [CrossRef] [PubMed]

11. Cassar, L. Photocatalysis of cementitious materials: Clean buildings and clean air. MRS Bull. 2004. [CrossRef]

12. Folli, A.; Pochard, I.; Nonat, A.; Jakobsen, U.H.; Shepherd, A.M.; Macphee, D.E. Engineering photocatalytic Cements: Understanding $\mathrm{TiO}_{2}$ surface chemistry to control and modulate photocatalytic performances. J. Am. Ceram. Soc. 2010, 939, 3360-3369. [CrossRef]

13. MacPhee, D.E.; Folli, A. Photocatalytic concretes-The interface between photocatalysis and cement chemistry. Cem. Concr. Res. 2016, 85, 48-54. [CrossRef] 
14. Folli, A.; Pade, C.; Hansen, T.B.; De Marco, T.; MacPhee, D.E. $\mathrm{TiO}_{2}$ photocatalysis in cementitious systems: Insights into self-cleaning and depollution chemistry. Cem. Concr. Res. 2012, 42, 539-548. [CrossRef]

15. Zhang, R.; Cheng, X.; Hou, P.; Ye, Z. Influences of nano- $\mathrm{TiO}_{2}$ on the properties of cement-based materials: Hydration and drying shrinkage. Constr. Build. Mater. 2015, 81, 35-41. [CrossRef]

16. Yang, L.; Wang, F.; Hakki, A.; Macphee, D.E.; Liu, P.; Hu, S. The influence of zeolites fly ash bead $/ \mathrm{TiO}_{2}$ composite material surface morphologies on their adsorption and photocatalytic performance. Appl. Surf. Sci. 2017, 392, 687-696. [CrossRef]

17. Hakki, A.; Yang, L.; Wang, F.; Macphee, D.E. The Effect of Interfacial Chemical Bonding in $\mathrm{TiO}_{2}$ Composites on Their Photocatalytic NOx Abatement Performance. J. Vis. Exp. 2017, 4. [CrossRef]

18. Yang, L.; Hakki, A.; Wang, F.; Macphee, D.E. Different Roles of Water in Photocatalytic DeNOx Mechanisms on $\mathrm{TiO}_{2}$ : Basis for Engineering Nitrate Selectivity? ACS Appl. Mater. Interfaces 2017, 9, 17034-17041. [CrossRef]

19. Fateh, R.; Dillert, R.; Bahnemann, D. Preparation and characterization of transparent hydrophilic photocatalytic $\mathrm{TiO}_{2} / \mathrm{SiO}_{2}$ thin films on polycarbonate. Langmuir 2013, 29, 3730-3739. [CrossRef]

20. Hanus, M.J.; Harris, A.T. Nanotechnology innovations for the construction industry. Prog. Mater. Sci. 2013, 58, 1056-1102. [CrossRef]

21. Lucas, S.S.; Ferreira, V.M.; De Aguiar, J.L.B. Incorporation of titanium dioxide nanoparticles in mortars-Influence of microstructure in the hardened state properties and photocatalytic activity. Cem. Concr. Res. 2013, 43, 112-120. [CrossRef]

22. Ballari, M.M.; Hunger, M.; Hüsken, G.; Brouwers, H.J.H. NOx photocatalytic degradation employing concrete pavement containing titanium dioxide. Appl. Catal. B Environ. 2010, 95, 245-254. [CrossRef]

23. ISO. ISO 22197-1: Fine Ceramics (Advanced Ceramics, Advanced Technical Ceramics)—Test Method for air-purification Performance of Semiconducting Photocatalytic Materials-Part 1: Removal of Nitric Oxide; ISO: Geneva, Switzerland, 2007.

24. Kisch, H.; Bahnemann, D. Best Practice in Photocatalysis: Comparing Rates or Apparent Quantum Yields? J. Phys. Chem. Lett. 2015, 6, 1907-1910. [CrossRef] [PubMed]

25. Freitag, J.; Domínguez, A.; Niehaus, T.A.; Hülsewig, A.; Dillert, R.; Frauenheim, T.; Bahnemann, D.W. Nitrogen(II) oxide charge transfer complexes on $\mathrm{TiO}_{2}$ : A new source for visible-light activity. J. Phys. Chem. C 2015, 119, 4488-4501. [CrossRef] 